\title{
Phase II Study of Bronchial Arterial Infusion of P53 Gene in Treatment of Multiple Lung Metastases of Hepatocellular Carcinoma
}

\author{
Wei Chen, Mei Li, Xingyu Fang, Qingsheng Fan and Miao Yu* \\ Department of Interventional Radiology, 301 Military General Hospital, Beijing 100853, China
}

\begin{abstract}
Objectives: To explore efficacy and safety of bronchial arterial infusion of recombinant adenovirus p53 gene (rAd-p53, or Gendicine ${ }^{\circledR}$ ) in treatment of multiple lung metastases tumor from Hepatocellular Carcinoma (HCC).

Methods: Thirty two patients with HCC and multiple pulmonary metastases were included in this study. Lung metastatic tumors were treated using bronchial arterial infusion of rAd-p53 via a transcatheter. Two infusions, once per week, were given to each lung area containing metastatic tumors at a dose of 2-4 $\times 10^{12}$ Virus Particles (VP) per infusion. All these patients were followed up every 3 month. Spiral CT was performed during follow-up visits to monitor tumor progress.

Results: At 3 months after the last treatment, 6 patients showed lung metastatic tumors completely disappeared, 15 patients showed a decrease in number of tumors, 2 patients un-changed in tumor number, and 9 patients developed new metastatic tumors. Overall survival rate of one year was $93.7 \%$ and median survival time was 18.4 months. There were no serious adverse events except for self-limited fever $\left(38^{\circ} \mathrm{C}-39.5^{\circ} \mathrm{C}\right)$ was found in 28 patients.
\end{abstract}

Conclusions: Bronchial arterial infusion of rAd-p53 is an effective and safe in treatment of multiple lung metastatic tumors of HCC.

Keywords: P53; Gene therapy; Hepatocellular carcinoma; Pulmonary metastasis

\section{Introduction}

Primary hepatocellular carcinoma (HCC) is the third leading causes of cancer-related death worldwide and its five-year survival rate is under $10 \%[1,2]$. The lungs are most common sites of metastasis of HCC and pulmonary metastatic rate of HCC is estimated around $45 \%$ [3]. After comprehensive treatment of HCC, the patients' survival time is prolonged but companied with a high rate of late pulmonary metastasis. Thus, treatment of pulmonary metastatic tumors may offer a chance for long-term survival. Surgical removal of pulmonary metastatic tumors is an option for small fraction of patients [4]. The most HCC patients with lung pulmonary metastases are not suitable for curative pulmonary metastatic tumor resection due to patients' poor condition or multiple metastases.

One of our previous reports demonstrated that p53 gene therapy delivered by intra-tumor injection with or without bronchial arterial infusion is effective to control pulmonary metastatic tumors with 1 or 2 metastatic tumor nodules from HCC [5]. In this report we show that bronchial arterial infusion of p53 gene therapy is an effective treatment for multiple diffuse pulmonary metastatic tumors.

\section{Methods}

\section{Patients}

From May 2009 to August 201132 HCC patients with multiple diffuse pulmonary metastatic tumors satisfying the following inclusion criteria were enrolled this study: histopathologically diagnosed HCC with multiple diffuse pulmonary metastatic tumors; with an Eastern Cooperative Oncology Group (ECOG) score of 0-2; with normal hemogram, blood coagulation, liver and kidney function tests; understanding and signing the informed consent form. The study was proved by our hospital ethics committee but not registered in an international clinical trial registry platform. There were 13 patients having pulmonary metastases at primary diagnosis and 19 patients developed pulmonary metastases. Patients' characteristics are summarized in Table 1.

\section{Treatments}

The rAd-p53 or Gendicine ${ }^{\circledR}$ was purchased from SiBiono GeneTech Co. Ltd (19 First Science \& technology Middle Road, Shenzhen, Guangdong 518057, P. R. China). The catheter was inserted to a bronchial artery through one femoral artery under Digital Subtraction Angiography (DSA) guidance. The contrast medium was injected to find the right arterial branch feeding tumors. If metastatic tumors spread to one whole lung the main bronchial artery is chosen. If the metastatic tumors spread to both lungs, the rAd-p53 was given to both sides. The rAd-p53 was diluted into $20 \mathrm{ml}$ physical saline and slowly injected into the chosen artery at a dose of $2-4 \times 10^{12}$ virus particle (VP) according to the artery supplying areas. Two infusions, once per week, were given to each lung areas containing metastatic tumors.

\section{Clinical evaluation}

All these patients were followed up every 3 month. Before p53 gene therapy and at each follow-up visit, all patients took routine blood test,

${ }^{*}$ Corresponding author: Miao Yu, Department of Interventional Radiology, 301 military general hospital, Beijing100853, China, E-mail: ggao1962@yahoo.com

Received May 02, 2012; Accepted May 18, 2012; Published May 21, 2012

Citation: Chen W, Li M, Fang X, Fan Q, Yu M (2012) Phase II Study of Bronchia Arterial Infusion of P53 Gene in Treatment of Multiple Lung Metastases of Hepatocellular Carcinoma. J Cancer Sci Ther 4: 124-126. doi:10.4172/1948 5956.1000125

Copyright: $\odot 2012$ Chen W, et al. This is an open-access article distributed unde the terms of the Creative Commons Attribution License, which permits unrestricted use, distribution, and reproduction in any medium, provided the original author and source are credited. 


\begin{tabular}{|l|l|}
\hline Characteristics & Statistics \\
\hline Age (years) & $54 \pm 31.5(40-82$ \\
\hline Gender & \\
\hline Male & $26(81.3 \%)$ \\
\hline Female & $6(18.7 \%)$ \\
\hline Number of metastatic nodules in lungs at treatment & \\
\hline 3 & $2(6.2 \%)$ \\
\hline $3-6$ & $11(34.4 \%)$ \\
\hline$\geq 6$ & $19(59.4 \%)$ \\
\hline Metastatic tumors in right or/and left lungs & \\
\hline Right & $11(34.4 \%)$ \\
\hline Left & $8(25.0 \%)$ \\
\hline Both & $13(40.6 \%)$ \\
\hline Primary HCC therapy & \\
\hline Surgical resection & $22(68.7 \%)$ \\
\hline Transcatheter arterial chemoembolisation & $7(21.9 \%)$ \\
\hline Percutaneous ethanol injection & $2(6.3 \%)$ \\
\hline Cryoablation & $1(3.1 \%)$ \\
\hline
\end{tabular}

Table 1: Patients' Characteristics.

liver and kidney function tests, as well as the chest and upper abdomen CT or MRI horizontal scan. The clinical symptoms such as cough, chest pain, abdominal pain, loss of appetite, fatigue, weight loss, etc. were evaluated. The survival time and cumulative survival rates were calculated using the Kaplan-Meier method. The progress-free survival time for pulmonary metastases was defined as the time between the day of starting the gene therapy and the day detecting progression of pulmonary metastases.

\section{Results}

Follow-up period after the last infusion ranged from 3.5 to 29 months with median of 16 months. At 3 months after treatment, 6 patients showed lung metastatic tumors completely disappeared, 15 patients showed a decrease in number of tumors, 2 patients un-changed in tumor number, and 9 patients developed new metastatic tumors. The treatment would be considered effective if the metastatic tumors disappeared, or number of metastatic tumors decreased, or number of metastatic tumors kept stable without new metastatic tumors for 4 weeks. Thus, the overall response rate was $23 / 32$ (71.9\%). Two patients died in 6 and 8 months after the last treatment as a result of progressed cancer with multiple organ failure. Overall survival rate of one year was $93.7 \%$ and median survival time was 18.4 months. The median progress-free survival time for pulmonary metastases was 15.8 months. Overall clinical symptoms alleviated in 25 patients (78.1\%). Figure 1 shows the CT images from a typical patient at the 3-month follow-up visit: metastatic tumors in the two lungs completely disappeared.

Mild to moderate fever occurred in 28 patients. The fever developed typically in 4-6 hours after the rAd-p53 infusion, and last for 8-20 hours. Other side effects included mild flu-like symptoms observed early in 7 patients and leukocytosis found in 3 patients. There were no serious adverse events was reported.

\section{Discussion}

Advances in local treatment modalities for primary HCC has improved local control and had a better prognosis. For further prolonging survival time, an effective treatment for extrahepatic metastases is needed. Pulmonary metastases account for over 50\% of extrahepatic HCC metastases [6,7]. Therefore, active treatments of pulmonary metastatic tumors may offer a chance for long-term survival. HCC is relatively resistant to both chemo- and radio-therapy. There were very few reports demonstrating a successful control of pulmonary metastases of HCC using chemo- or radio-therapy $[8,9]$. The HCC patients with distant metastases are usually in poor general condition, and can't stand the side effects of chemo- or radio-therapy. Surgery currently offers the best probability for cure and local control of pulmonary metastases. Kawamura $\mathrm{M}$ analyzed the results of 61 patients after surgical resection for pulmonary metastasis from HCC and showed overall 5-year survival rate of $32.2 \%$ [10]. Lam et al. [11] reported a 5 -year survival rate of $67 \%$ in patients who underwent resection of solitary pulmonary metastatic tumor. Lee et al. [12] indicated that surgical resection for pulmonary metastasis from HCC might be beneficial for patients whose HCC was controlled by surgery and the number of pulmonary metastatic tumors were lower than 3 . However, the most HCC patients with lung pulmonary metastases are not suitable for curative pulmonary metastatic tumor resection due to patients' poor condition or multiple metastases. One of our previous reports demonstrated that $\mathrm{p} 53$ gene therapy delivered by intra-tumor injection with or without bronchial arterial infusion was effective to control pulmonary metastatic tumors with 1 or 2 metastatic tumor nodules from HCC [5]. This report shows that bronchial artery infusion of rAd-p53 can effectively control the multiple pulmonary metastases of HCC. The response rate was $71.9 \%$ and the lung metastatic tumors completely disappeared in 6 patients. Overall survival rate of one year was $93.7 \%$ and median survival time was 18.4 months. A longer period of follow-up time is needed to determine the long term efficacy results.

P53's antitumor functions and the clinical results using rAd-p53 in treatment of solid malignant tumors were well described in our previous report and by others [5,13]. Several reports demonstrated

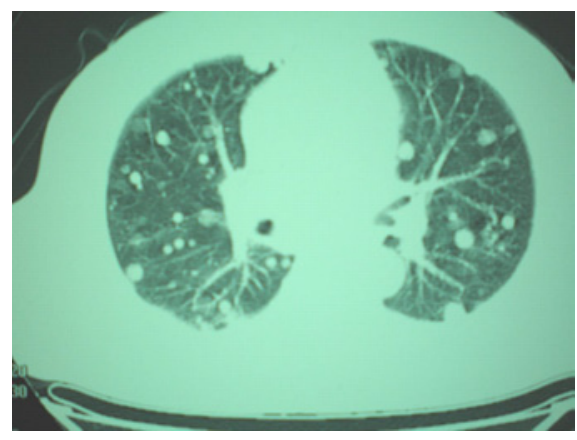

Figure 1a: A CT scan before treatment showed : multiple metastatic tumors in both lungs.

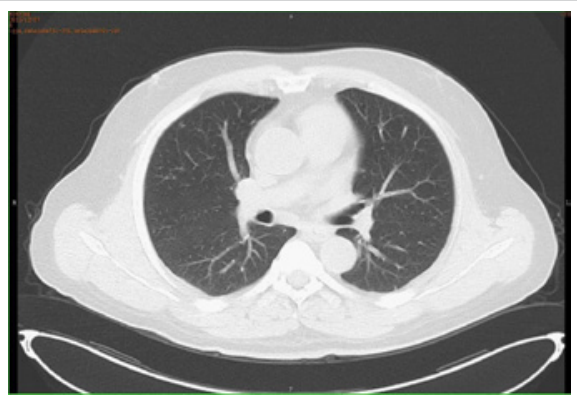

Figure 1b: A CT scan taken 6 months after treatment showed : metastatic tumors completely disappeared. 
that rAd-p53 combined with transcatheter arterial chemoembolization or transcatheter arterial embolisation were very effective treatment for primary HCC [14-16]. Tian et al. [17] suggested that p53 gene has a strong inhibitory activity against hepatic tumor cells. The Inhibitory effect was positively correlated with the percentage of transfected cells. This report and our previous reports demonstrated that rAd-p53 can effectively control the pulmonary metastases of HCC. These data indicates rAd-p53 alone or combined with other therapies is an appropriate treatment for HCC and pulmonary metastases of HCC.

We did not investigate the p53 mutation status in HCC. According to reports, the p53 mutation rate in HCC is around 50\% [18-20]. Our response rates from previous and this study were $83.3 \%$ [5] and $71.9 \%$, respectively, which is much higher than the reported p53 mutation rate. These results indicate that the HCC response to p53 gene therapy is not strictly associated with the p53 mutation status. We are conducting a phase II study to investigate the relationship between the p53 mutation status in HCC in advanced stage and the HCC response rate to p53 gene therapy.

In this study, we did not examine the p53 and its downstream gene expression in the tumor tissues. According Tian's [16] report, 48 hours after the first hepatic arterial injection of rAd-p53, vector specific p53 mRNA expression in HCC tissue was positive in 12 of 15 patients (80\%). After multiple injections, all the 15 patients showed high expression of vector specific $\mathrm{p} 53$ and $\mathrm{p} 21 \mathrm{mRNA}$.

Another advantage to choose rAd-p53 for treatment of pulmonary metastases of HCC is its high safety profile. The side effects of rAd-p53 are mild, and mild to medium self-limited fever is mostly reported. Since it was on marketing, there have been no serious adverse events observed. The HCC patients with extraheptic metastases are usually in poor general condition and they can't stand conventional treatments such as surgery, chemo- or radiotherapy. Thus, rAd-p53 gene therapy is an appropriate choice for these patients.

In summary, bronchial artery infusion of rAd-p53 gene therapy is an effective and appropriate treatment for the multiple pulmonary metastases of HCC.

\section{References}

1. Murugavel KG, Naranatt PP, Shankar EM, Mathews S, Raghuram K et al. (2007) Prevalence of aflatoxin B1 in liver biopsies of proven hepatocellular carcinoma in India determined by an in-house immunoperoxidase test. J Med Microbiol 56: 1455-1459.

2. Tang ZY (2006) Significance and approaches of carrying out research on metastasis and recurrence of liver cancer. Chin J Gen Surg 21: 761-764.

3. Ikai I, Itai Y, Okita K, Omata M, Kojiro M, et al. (2004) Report of the 15th followup survey of primary liver cancer. Hepatol Res 28: 21-29.

4. van Geel AN, Pastorino U, Jauch KW, Judson IR, van Coevorden F, et al. (1996) Surgical treatment of lung metastases: The European Organization for Research and Treatment of Cancer-Soft Tissue and Bone Sarcoma Group study of 255 patients. Cancer 77: 675-682.

5. Yu M, Chen W, Zhang J (2010) p53 gene therapy for pulmonary metastasis tumor from hepatocellular carcinoma. Anticancer Drugs 21: 882-884.

6. Natsuizaka M, Omura T, Akaike T, Kuwata Y, Yamazaki K, et al. (2005) Clinical features of hepatocellular carcinoma with extrahepatic metastases. J Gastroenterol Hepatol 20: 1781-1787.

7. Katyal S, Oliver JH, Peterson MS, Ferris JV, Carr BS, et al. (2000) Extrahepatic metastasis of hepatocellular carcinoma. Radiology 216: 698-703.

8. Nakamura M, Nagano H, Marubashi S, Miyamoto A, Takeda Y, et al. (2008)
Pilot study of combination chemotherapy of S-1, a novel oral DPD inhibitor and interferon-alpha for advanced hepatocellular carcinoma with extrahepatic metastasis. Cancer 112: 1765-1771.

9. Ishikawa T, Ichida T, Yokoyama J, Matsuda Y, Watanabe T, et al. (2004) Complete disappearance of pulmonary metastases in a case of hepatocellular carcinoma treated with docetaxel-based systemic chemotherapy. J Gastroenterol Hepatol 19: 1423-1426.

10. Kawamura M, Nakajima J, Matsuguma H, Horio H, Miyoshi S, et al. (2008) Surgical outcomes for pulmonary metastases from hepatocellular carcinoma. Eur J Cardiothorac Surg 34: 196-199.

11. Lam CM, Lo CM, Yuen WK, Liu CL, Fan ST (1998) Prolonged survival in selected patients following surgical resection for pulmonary metastasis from hepatocellular carcinoma. Br J Surg 85: 1198-1200.

12. Lee CY, Bae MK, Park IK, Kim DJ, Lee JG, et al. (2010) Surgical resection for pulmonary metastasis from hepatocellular carcinoma: analysis of prognosis in relation to primary control. J Surg Oncol 101: 239-243.

13. Pan JJ, Zhang SW, Chen CB, Xiao SW, Sun Y, et al. (2009) Effect of recombinant adenovirus-p53 combined with radiotherapy on long-term prognosis of advanced nasopharyngeal carcinoma. J Clin Oncol 27: 799-804.

14. Guan YS, Liu Y, Zhou XP, Li X, He Q, et al. (2005) p53 gene (Gendicine) and embolisation overcame recurrent hepatocellular carcinoma. Gut 54: 13181319.

15. Tian G, Liu J, Sui J (2009) A patient with huge hepatocellular carcinoma who had a complete clinical response to p53 gene combined with chemotherapy and transcatheter arterial chemoembolization. Anticancer Drugs 20: 403-407.

16. Tian G, Liu J, Zhou JS, Chen W (2009) Multiple hepatic arterial injections of recombinant adenovirus p53 and 5-fluorouracil after transcatheter arterial chemoembolization for unresectable hepatocellular carcinoma: a pilot phase II trial. Anticancer Drugs 20: 389-395.

17. Tian G, Liu JL, Dong HS (2007) Experimental study of p53 gene on the inhibition of human's hepatocellular carcinoma cells. Shengzhen Chinese and Western Medicine Integration Magazine 17: 286-290.

18. Hsu H, Peng S, Lai P, Chu J, Lee P (1994) Mutations of p53 gene in hepatocellular-carcinoma (hcc) correlate with tumor progression and patient prognosis - a study of 138 patients with unifocal hcc. Int J Oncol 4: 1341-1347.

19. Oda T, Tsuda H, Scarpa A, Sakamoto M, Hirohashi S (1992) Mutation pattern of the p53 gene as a diagnostic marker for multiple hepatocellular carcinoma. Cancer Res 52: 3674-3678.

20. Hsu IC, Metcalf RA, Sun T, Welsh JA, Wang NJ, et al. (1991) Mutational hotspot in the p53 gene in human hepatocellular carcinomas. Nature 350: 427-428. 